\title{
A short-duration event as the cause of dust ejection from Main-Belt Comet P/2012 F5 (Gibbs)
}

\author{
F. Moreno \\ Instituto de Astrofísica de Andalucía, CSIC, Glorieta de la Astronomía s/n, 18008 \\ Granada, Spain \\ fernando@iaa.es \\ J. Licandro \\ Instituto de Astrofísica de Canarias, c/Vía Láctea s/n, 38200 La Laguna, Tenerife, Spain, \\ and \\ Departamento de Astrofísica, Universidad de La Laguna (ULL), E-38205 La Laguna, \\ Tenerife, Spain \\ and \\ A. Cabrera-Lavers \\ Instituto de Astrofísica de Canarias, c/Vía Láctea s/n, 38200 La Laguna, Tenerife, Spain, \\ and \\ Departamento de Astrofísica, Universidad de La Laguna (ULL), E-38205 La Laguna, \\ Tenerife, Spain, \\ and \\ GTC Project, E-38205 La Laguna, Tenerife, Spain
}

\begin{abstract}
We present observations and an interpretative model of the dust environment of Main-Belt Comet P/2010 F5 (Gibbs). The narrow dust trails observed can be interpreted unequivocally as an impulsive event that took place around 2011 July 1st with an uncertainty of \pm 10 days, and a duration of less than a day, possibly of the order of a few hours. The best Monte Carlo dust model fits to the observed trail brightness imply ejection velocities in the range $8-10 \mathrm{~cm} \mathrm{~s}^{-1}$ for particle sizes between $30 \mathrm{~cm}$ and $130 \mu \mathrm{m}$. This weak dependence of velocity on size contrasts with that expected from ice sublimation, and agrees with that found recently for (596) Scheila, a likely impacted asteroid. The particles seen in the trail are found
\end{abstract}


to follow a power-law size distribution of index $\approx-3.7$. Assuming that the slowest particles were ejected at the escape velocity of the nucleus, its size is constrained to about $200-300 \mathrm{~m}$ in diameter. The total ejected dust mass is $\gtrsim 5 \times 10^{8} \mathrm{~kg}$, with represents approximately 4 to $20 \%$ of the nucleus mass.

Subject headings: Minor planets, asteroids: individual (P/2012 F5 (Gibbs) — Methods: numerical

\section{Introduction}

Main-Belt Comet P/2012 F5 (Gibbs) (hereafter P/Gibbs) was discovered in the course of the Mt. Lemmon Survey on UT 2012 Mar. 22.89 (Gibbs et al. 2012). The object showed a narrow dust tail $7^{\prime}$ in length, and was identified as a Main-Belt Comet (MBC) owing to its orbital parameters. MBCs have dynamical properties of asteroids (i.e., Tisserand parameters respect to Jupiter larger than 3), but physical characteristics of comets (gas and/or dust emission). The total members of this new class of Small Solar System Objects amount to nine (the 9th becoming $\mathrm{P} /$ Gibbs), so that the statistics is still poor so as to identify their global physical properties or dynamical history. For reviews on those objects, we refer to Bertini (2011) and Jewitt (2012). Most of the MBCs have been shown to be dynamically stable on timescales of $100 \mathrm{Myr}$ or longer (Ipatov \& Hahn 1999; Hsieh et al. 2012a,b.,c; Stevenson et al. 2012) suggesting that they are native members of the Main Asteroid Belt and not captured objects from elsewhere (Hsieh et al. 2009). This is strongly supported by dynamical and spectroscopic arguments that show that some of them belong to well established asteroid collisional families and that their spectra is different to that of "normal" comets (e.g. Licandro et al. 2011). However, there are some members as 238P/Read and P/2008 R1 that are stable for 20-30 Myr only (Haghighipour 2009; Jewitt et al. 2009). Regarding their activity, some are clearly recurrent, as 133P/Elst-Pizarro and 238P (Hsieh et al. 2010, 2011), while some others like (596) Scheila appear to have ejected dust via an impulsive event that might be associated to a collision (Jewitt et al. 2011; Yang \& Hsieh 2011; Bodewits et al. 2011; Moreno et al. 2011a; Ishiguro et al. 2011). On the other hand, P/2010 R2 (La Sagra) and 2006 VW139 appeared to be continuously active during periods of at least 200 days and 100 days, respectively (Moreno et al. 2011b; Licandro et al. 2012; Hsieh et al. 2012b).

Observations and analysis of $\mathrm{P} /$ Gibbs have been recently shown by Stevenson et al. (2012), who report on the impulsive character of the emission of the dust, the outburst date, the mass of the ejecta, and establish limits to the nucleus size. In this paper we report images in the red spectral domain acquired with instrumentation attached to the 10.4-m Gran Telescopio Canarias (GTC) of P/Gibbs at two different dates, and perform an 
interpretation of the observed trail brightness using a forward Monte Carlo tail model, in order to provide estimates of the dust emission times, the ejected mass, the particle ejection velocities, and their size distribution function. We also set limits to the nucleus size based on the escape velocity, and compare our results to those by Stevenson et al. (2012).

\section{Observations and data reduction}

CCD images of $\mathrm{P} /$ Gibbs were collected under photometric conditions on the nights of 18 May and 8 June 2012, using a Sloan $r^{\prime}$ filter in the Optical System for Image and Low Resolution Integrated Spectroscopy (OSIRIS) camera-spectrograph (Cepa et al. 2000; Cepa 2010) at the GTC. The OSIRIS instrument consists of two Marconi CCD detectors, each with $2048 \times 4096$ pixels and a total unvignetted field of view of $7.8^{\prime} \times 7.8^{\prime}$. The plate scale was $0.127 " / \mathrm{px}$, but we used a binning of $2 \times 2$ pixels in order to improve the signal to noise ratio, so that the spatial resolution of the images is $485 \mathrm{~km} \mathrm{px}^{-1}$ and $540 \mathrm{~km} \mathrm{px}^{-1}$ on May 18.9 and June 8.9, respectively. The images were bias subtracted and flat-field corrected using standard procedures. A total of 18 images were acquired each night and calibrated using standard stars. The images were converted to solar disk intensity units appropriate for the analysis in terms of dust tail models, and a median stack image was obtained for analysis for each date (see Figure 1). As a result of both the flux calibration and the median stacking procedure, we estimated a total flux uncertainty in the combined images of $0.3 \mathrm{mag}$. The images were finally rotated to the $(N, M)$ coordinate system (Finson \& Probstein 1968).

\section{The Model}

We have performed an analysis of the two images shown in Figure 1 by a direct Monte Carlo dust tail model. This model was used to characterize the dust environments of various comets and MBCs (e.g., Moreno 2009; Moreno et al. 2010, 2011b), being applied in particular to comet $67 \mathrm{P} /$ Churyumov-Gerasimenko, the target of Rosetta Mission to arrive to the comet in 2014 (the so-called Granada model, see Fulle et al. 2010). The code is used to compute the trajectory of a large number of grains ejected from a cometary or asteroidal surface. We assume that the only governing forces on those dust particles are the solar gravity and the radiation pressure. Thus, the gravity of the object itself is neglected, which constitutes a good approximation for small-sized nuclei. In the case of cometary activity, we consider that the particles are accelerated by gas drag from ice sublimation to their terminal velocities, which are the input ejection velocities considered in the model, that is applicable for any

other ejection mechanism. Once ejected, the particles describe a Keplerian trajectory around 
the Sun, whose orbital elements are computed from the terminal velocity and the ratio of the force exerted by the solar radiation pressure and the solar gravity (the $\beta$ parameter, see Fulle 1989). This parameter can be expressed as $\beta=C_{p r} Q_{p r} /(2 \rho r)$, where $C_{p r}=1.19 \times$ $10^{-3} \mathrm{~kg} \mathrm{~m}^{-2}, Q_{p r}$ is the radiation presure coefficient, and $\rho$ is the particle density. For each observation date, the trajectories of a large number of dust particles are computed, and then their positions on the $(N, M)$ plane are calculated. Finally, their contribution to the tail brightness is computed.

Before applying the model, we performed a preliminary analysis of the images in terms of a synchrone map (Finson \& Probstein 1968), which provides us with an approximate idea on the time interval at which the particles were ejected from the nucleus related to the observation date. Figure 2 shows the two images as well as the corresponding synchrones at times of $\pm 100, \pm 20, \pm 10$ and 0 days relative to the 2011 July 3rd synchrone, the one which is approximately best aligned with the narrow trail in each image. The smallest sized particles in the 2011 July 3rd synchrones have $r \sim 130 \mu \mathrm{m}$ and $r \sim 180 \mu \mathrm{m}$ for the two observing dates, respectively. These diagrams indicate that all the dust particles having $r \gtrsim 130 \mu \mathrm{m}$ were ejected during a very short time interval around that date, because otherwise the trails would have been wider towards the lowermost portions of the trails, even if they were ejected with zero velocity respect to the nucleus. Therefore, an impulsive event must be the responsible of the ejection of dust, so that an outburst, a collision with another body, or a rotational disruption could be in principle invoked among the responsible mechanisms. The precise event date, and its duration, is to be determined on the basis of the Monte Carlo dust tail analysis (see section 4).

An important aspect of the observations and the modeling is the location of the asteroid nucleus. We will work under the assumption that the object nucleus is immersed in the dust cloud, and that the optocenter of this cloud corresponds to the actual location of the nucleus. We further assume that the observed brightness is dominated by the dust, the contribution of the nucleus being negligible. This hypothesis would be tested on the basis of the nucleus size derived from arguments based on the escape velocity.

A number of simplifying assumptions on the physical parameters must be made in order to make the problem tractable. Thus, we assume that the trail is composed of spherical particles of carbonaceous composition, having a refractive index at red wavelengths of $\mathrm{m}=1.88+0.71 i($ Edoh 1983$)$, which implies a geometric albedo of $p_{v}=0.036$, and a pressure radiation coefficient of $Q_{p r} \sim 1$ for particles of radius $r \gtrsim 1 \mu \mathrm{m}$ (Moreno et al. 2012, their Figure 5). We further assume that the particles have a density of $1000 \mathrm{~kg} \mathrm{~m}^{-3}$. The particle size distribution is assumed to follow a power-law of index $\alpha$, and the terminal velocities are described as a function of the $\beta$ parameter as $v(\beta)=v_{0} \beta^{\gamma}$. This relationship is generally 
accepted for the terminal velocities of comet dust, and also for fragments ejected from collision experiments (e.g., Giblin 1998; Onose \& Fujiwara 2004). The total ejected dust mass $\left(M_{e}\right)$ and the maximum size of the ejected particles $\left(r_{\max }\right)$ must be also specified, as well as the starting time $\left(t_{s}\right)$ and the duration of the event $(\Delta t)$. In summary, there are a total of seven adjustable parameters to fit the observations $\left(\alpha, v_{0}, \gamma, M_{e}, r_{\max }, t_{s}\right.$, and $\left.\Delta t\right)$.

\section{Results}

We attempted to fit the observed dust trail brightness by minimizing the function $\sigma=$ $\sigma_{1}+\sigma_{2}$, where the subscripts 1 and 2 correspond to the images obtained in 2012 May 18.9 and 2012 June 8.9, respectively, and $\sigma_{i}=\sqrt{\left(\sum\left(I_{o b s}(i)-I_{f i t}(i)\right)^{2} / N(i)\right)}$, where $I_{o b s}(i)$ are the observed trail intensities and $I_{f i t}(i)$ are the fitted intensities, the summation being extended in principle to all the image pixels $N(i)$. Since there are some regions in the images that are strongly contaminated by bright field stars, we restricted the summation to pixels outside those regions, and located mostly along the trails. The minimization procedure was performed by the multidimensional downhill simplex algorithm (Nelder \& Mead 1965), using the FORTRAN implementation described in Press et al. (1992). Each of those parameters influence the derived trail brightness in different ways. Thus, $v_{0}$ controls the width of the trail, $\gamma$ influences the variation of the width of the trail along it, $\alpha$ constraints the slope of the brightness along the trail, the total ejected mass influences the overall brightness, and the maximum particle size controls the brightness mainly at the head of the trail, so that

if $r_{\text {max }}$ is set to a small value (say, smaller than several centimeters) the peak of brightness is displaced significantly trailward and the fit becomes impossible. Regarding the minimum particle size, it is not a free parameter, as it is found by the intersection of the synchrone that is best aligned with the trails and the $N$ axis, which corresponds in our images to $\sim 130$ $\mu \mathrm{m}$ and $\sim 180 \mu \mathrm{m}$ for 2012 May 18.9 and 2012 June 8.9, respectively.

Since the downhill simplex method searches for a local minimum of the function in the parameter space, we performed several runs by varying the starting simplex. We found that all runs tend to converge to close local minima, giving similar values of $\sigma$. The deepest global minimum was then taken as the best fit, which has $\sigma=4.9 \times 10^{-15}$ solar disk units (see Table 1, and Figure 3). Unfortunately, this technique does not provide any estimate of the uncertainty in the derived parameters. To estimate those uncertainties, we determined the errors by perturbing the observed image intensities by the flux uncertainties and then finding a new best fit. In addition, we verified that outside the error limits for each one of the fitted parameters displayed in Table 1, no satisfying solutions were found. To do that, we tried to fit the model with values outside the parameter bounds specified in the table, and could not 
find satisfying solutions in any case.

An important result is that the derived ejection velocities are almost independent on size $\left(\gamma=0.04_{-0.02}^{+0.06}\right)$, ranging from about 10 to $8 \mathrm{~cm} \mathrm{~s}^{-1}$ for particles between the lower and upper size limits $(130 \mu \mathrm{m}$ and $28 \mathrm{~cm})$. This flat dependence of ejection velocities on size have been previously inferred in our analysis of the outburst of the asteroid (596) Scheila, for which we found $\gamma=0.05$ (Moreno et al. 2011a) . This dependence is markedly different to that expected from gas drag by ice sublimation processes as occur in most comets, with typical values of $\gamma \sim 0.5$, and could be associated to a collision event as was suggested for Scheila (Moreno et al. 2011a), although it cannot be confirmed. On the other hand, if we equate the velocity of the slowest moving particles $\left(v=8 \mathrm{~cm} \mathrm{~s}^{-1}\right.$ for $r=28 \mathrm{~cm}$ particles) to the escape velocity, we obtain a nucleus radius of $R_{n}=107 \mathrm{~m}$ to $R_{n}=152 \mathrm{~m}$, considering bulk densities of 1000 to $500 \mathrm{~kg} \mathrm{~m}^{-3}$. At this point, it is interesting to note that computer simulations at the catastrophic disruption threshold reveal that ejecta velocities depend on target size and that ejection velocities of the order of those obtained here $\left(\sim 10 \mathrm{~cm} \mathrm{~s}^{-1}\right)$ could be compatible with both porous and non-porous targets of $\sim 100 \mathrm{~m}$ radius (Jutzi et al. 2010). Our derived target radius is a far more stringent constraint than that of $R_{n}<2.1$ $\mathrm{km}$ derived by Stevenson et al. (2012) based on the non-detection of the nucleus on images taken by the Wide-Field Infrared Survey Explorer (WISE). On the other hand, using the formalism by Bowell et al. (1989), and assuming a slope parameter of $G=0.15$, and a bulk geometric albedo of $p_{v}=0.15$, the apparent magnitude of a $R_{n}=125 \mathrm{~m}$ nucleus at the same phase angle and geocentric and heliocentric distances than P/Gibbs would be just above $m_{v}=26$, which represents a negligible contribution to the brightness compared with that at the optocenter of the trails. This is compatible with our initial hypothesis on that the dust cloud brightness dominates the trail optocenter. An object of such a small size might have a very small rotation period that could be under the critical rotation period for fracture so that a rotational disruption could also be argued as the cause of the event (Jewitt 2012), provided it produces a sudden release of material. A small-sized nucleus was also derived for P/2010 A2 (LINEAR), the innermost MBC discovered so far, whose tail has been also reported by many authors as the result of an impulsive event (e.g. Jewitt et al. 2010; Snodgrass et al. 2010; Hainaut et al. 2012), and not as the result of a sustained activity as we suggested (Moreno et al. 2010).

The size distribution power index, which essentially controls the slope of the trail brightness, is $-3.7 \pm 0.1$. The maximum ejected particle size derived is $r=28 \pm 10 \mathrm{~cm}$ (Table 1 ). A maximum size smaller than the lower bound would imply a significant displacement of the maximum of brightness trailward. Conversely, a size larger than the upper bound would make the slope of the synthetic trail much steeper than observed. 
The minimum ejected dust mass is $(5 \pm 2) \times 10^{8} \mathrm{~kg}$, which is near the value reported by Hainaut et al. (2012) for P/2010 A2 $\left(8 \times 10^{8} \mathrm{~kg}\right)$. This is an order of magnitude higher than reported by Stevenson et al. (2012), although their result is just based on a measure of the total geometric cross section of the trail and assuming a mean size for the ejected particles, not a size distribution. This total mass is in fact a lower limit, since we cannot precise neither the amount of small $(r<100 \mu \mathrm{m})$ particles released, nor the component of particles traveling at high speed that could result as consequence of e.g. a collision, if this were the case.

The best-fit event date (2011 July 1st) agrees with that derived by Stevenson et al. (2012) within the error limits (they derived 2011 July 7 th as the event date). The duration of the event is constrained to be of less than a day. In the $\Delta t<1$ day space, it is always possible to find a set of fitting parameters giving similar values of $\sigma$. The fits shown in Figure 3 correspond to an event duration of 0.13 day, i.e., just above 3 hours. An impulsive event is, then, clearly implied.

\section{Conclusions}

From the Monte Carlo dust tail modeling of the observations of Main-Belt Comet P/2012 F5 (Gibbs) we can derive the following conclusions:

1) The direct study of the MBC images in term of synchrone analysis imply an impulsive event as the cause of the observed trails. We predict the event to have occurred on 2011 July 1st, with an accuracy of \pm 10 days. In this respect, we agree with the results recently

reported by Stevenson et al. (2012) for this MBC, not only in the nature, but also in the event date within the errors (they report on a impulsive event on 2011 July 7 th with an uncertainty of \pm 20 days). The duration of the event is constrained to be less than 1 day, possibly less than a few hours.

2) The nature of the impulsive event is impossible to determine with the sole information of the physical parameters derived from this analysis. Among the likely causes, an outburst, a collision with another body, or a rotational disruption could be invoked. Activity related to ice sublimation seems unlikely on the basis of the dependence of the particle ejection velocities on size, which interestingly turns out to be very similar to that we found for (596) Scheila (Moreno et al. 2011a), a very likely impacted asteroid.

3) The total dust mass released is $\gtrsim 5 \times 10^{8} \mathrm{~kg}$. The ejected particles are distributed in size following a power-law of index $-3.7 \pm 0.1$. The maximum particle size ejected is about $30 \mathrm{~cm}$, with a velocity of $\sim 8 \mathrm{~cm} \mathrm{~s}^{-1}$. Adopting this value as the escape velocity, the size of 
the nucleus is constrained to about $100-150 \mathrm{~m}$ in radius, for bulk body densities in the range $1000-500 \mathrm{~kg} \mathrm{~m}^{-3}$.

This article is based on observations made with the Gran Telescopio Canarias (GTC), installed in the Spanish Observatorio del Roque de los Muchachos of the Instituto de Astrofísica de Canarias, in the island of La Palma.

We gratefully acknowledge the comments and suggestions of an anonymous referee.

This work was supported by contracts AYA2009-08190, AYA2011-30613-C02-01, and FQM-4555 (Proyecto de Excelencia, Junta de Andalucía). J. Licandro gratefully acknowledges support from the Spanish "Ministerio de Ciencia e Innovación" project AYA201129489-C03-02.

\section{REFERENCES}

Bertini, I. 2011, Planet. Space Sci., 59, 365

Bodewits, D., Kelley, M.S., Li, J.Y., et al. 2011, ApJ, 733, L3

Bowell, E., Hapke, B., Domingue, D. et al., 1989, in Asteroids II, ed. R.P. Binzel, T. Gehrels, and M.S. Matthews, Univ. of Arizona Press, Tucson, p. 524.

Cepa, J., Aguiar, M., Escalera, V. et al. 2000, Poc. SPIE, 4008, 623

Cepa, J. 2010, Highlights of Spanish Astrophysics V, Astrophysics and Space Science Proceedings, Springer-Verlag, p. 15

Edoh, O. 1983, PhD thesis, Univ. Arizona

Finson, M., \& Probstein, R. 1968, ApJ, 154, 327

Fulle, M., 1989, A\&A, 217, 283

Fulle, M., Colangeli, L., Agarwal, J., et al. 2010, A\&A, 522, 63

Gibbs, A.R., Sato, H., Ryan, W.H. et al. 2012, Central Bureau Electronic Telegrams, 3069, 1

Giblin, I. 1998, Planet. Space Sci., 46, 921

Haghighipour, N. 2009 Meteor. \& Planet. Sci., 44, 1863 
Hainaut, O.R., Kleyna, J., Sarid, G. et al. 2012, A\&A 537, A69

Hsieh, H.H., Jewitt, D., \& Fernández, Y. 2004, AJ, 127, 2997

Hsieh, H.H., \& Jewitt, D. 2006, Science, 312, 561

Hsieh, H.H., \& Jewitt, D., \& Ishiguro, M. 2009, AJ, 137, 157

Hsieh, H.H., \& Jewitt, D., Lacerda, P. 2010, MNRAS, 403, 363

Hsieh, H.H., Meech, K., \& Pittichova, J. 2011b, ApJ, 736, L18

Hsieh, H.H., Yang, B., \& Haghighipour, N. 2012, ApJ, 744, 9

Hsieh, H.H., Yang, B., Haghighipour, N., et al. 2012, ApJ, 748, L15

Hsieh, H.H., Yang, B., Haghighipour, N., et al. 2012, AJ, 143, 104

Ipatov, S.I., \& Hahn, G.J. 1999, Solar Syst. Res. , 33, 487

Ishiguro, M., Hanayama, H., Hasegawa, S. et al. 2011, ApJ, 740, L11

Jewitt, D., Yang, B., \& Haghighipour, N. 2009 AJ, 137, 4313

Jewitt, D., Weaver, H., Agarwal, J., Mutchler, M., \& Drahus, M. 2010 Nature, 467, 817

Jewitt, D., Weaver, H., Mutchler, M., et al. 2011 ApJ, 733, L4

Jewitt, D. 2012 AJ, 143, 21

Jutzi, M., Michel, P., Benz, M., \& Richardson, B.C. 2010 Icarus, 207, 54

Licandro, J., Campins, H., Tozzi, G.P., et al. 2011 A\&A 532, 65

Licandro, J., de León, J., Moreno, F., et al. 2012 A\&A, submitted

Moreno, F. 2009, ApJS, 183, 33

Moreno, F., Licandro, J., Tozzi, G.-P., et al. 2010, ApJ, 718, L132

Moreno, F., Licandro, J., Ortiz, J.L., et al. 2011a, ApJ, 738, 130

Moreno, F., Lara, L.M., Licandro, J., et al. 2011b, ApJ, 738 L16

Moreno, F., Pozuelos, F., Aceituno, F., et al. 2012, ApJ, 752, 136

Nelder, J. A., \& Mead, R. 1965, Comput. J., 7, 308 
Onose, N., \& Fujiwara, A. 2004, Meteoritics and Planet. Sci., 39, 321

Press, W.H., Teukolsky, S.A., Vetterling, W.T., \& Flannery, B.P. 1992, in Numerical Recipes in FORTRAN (Cambridge: Cambridge Univ. Press), 402

Snodgrass, C., Tubiana, C., Vincent, J.-B. et al. 2010 Nature, 467, 814

Stevenson, R., Kramer, E.A., Bauer, J.M. et al. 2012, ApJ, in press

Yang, B., \& Hsieh, H.H., 2011, ApJ, 737, L39 

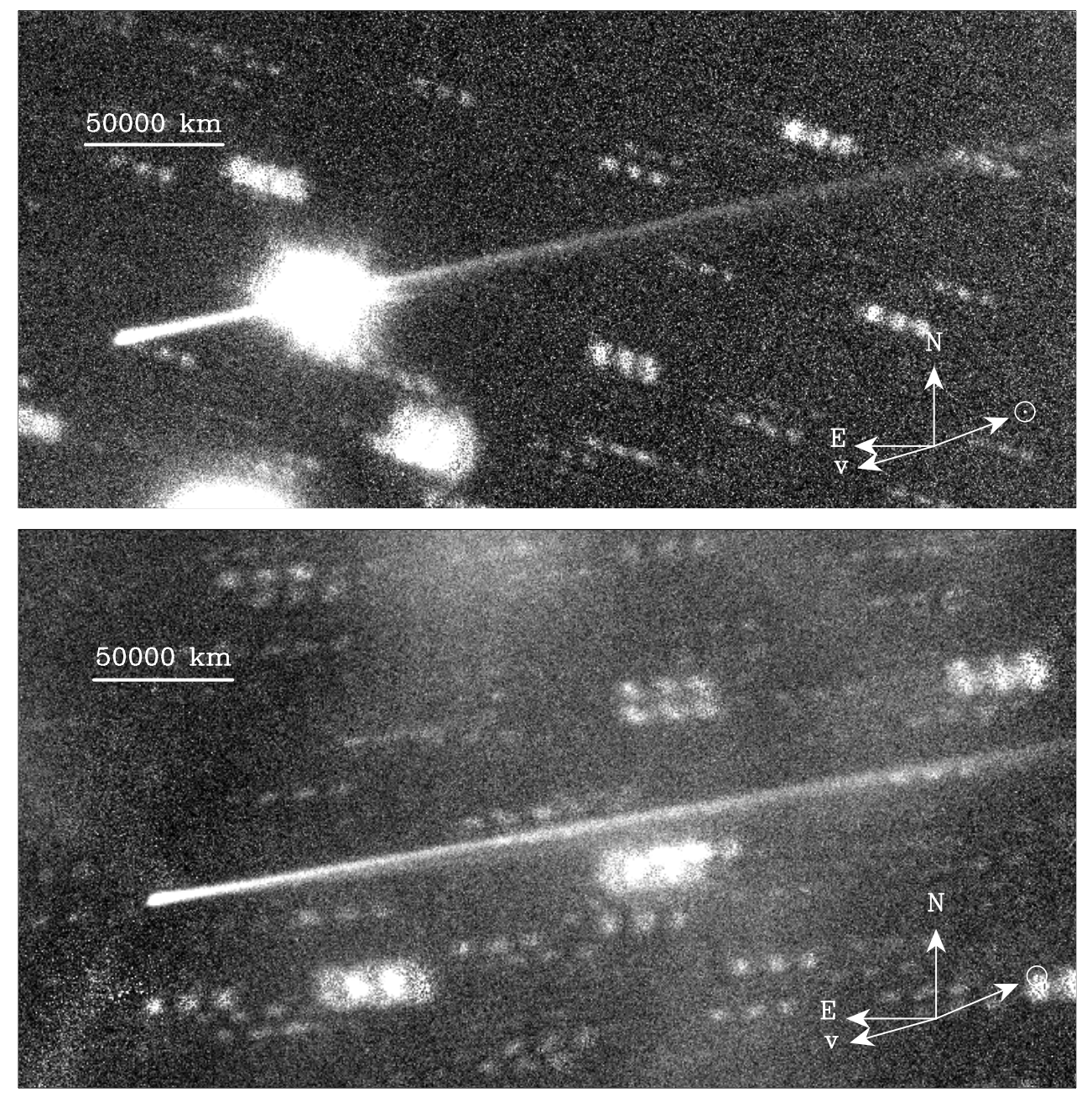

Fig. 1.- Median stack images of P/2012 F5 (Gibbs) obtained with the OSIRIS instrument of the 10.4m Gran Telescopio Canarias through a Sloan $r^{\prime}$ filter, on UT 2012 May 18.9 (upper panel) and June 8.9 (lower panel). The directions of the velocity vector, the Sun, and the astronomical North and East are indicated. 

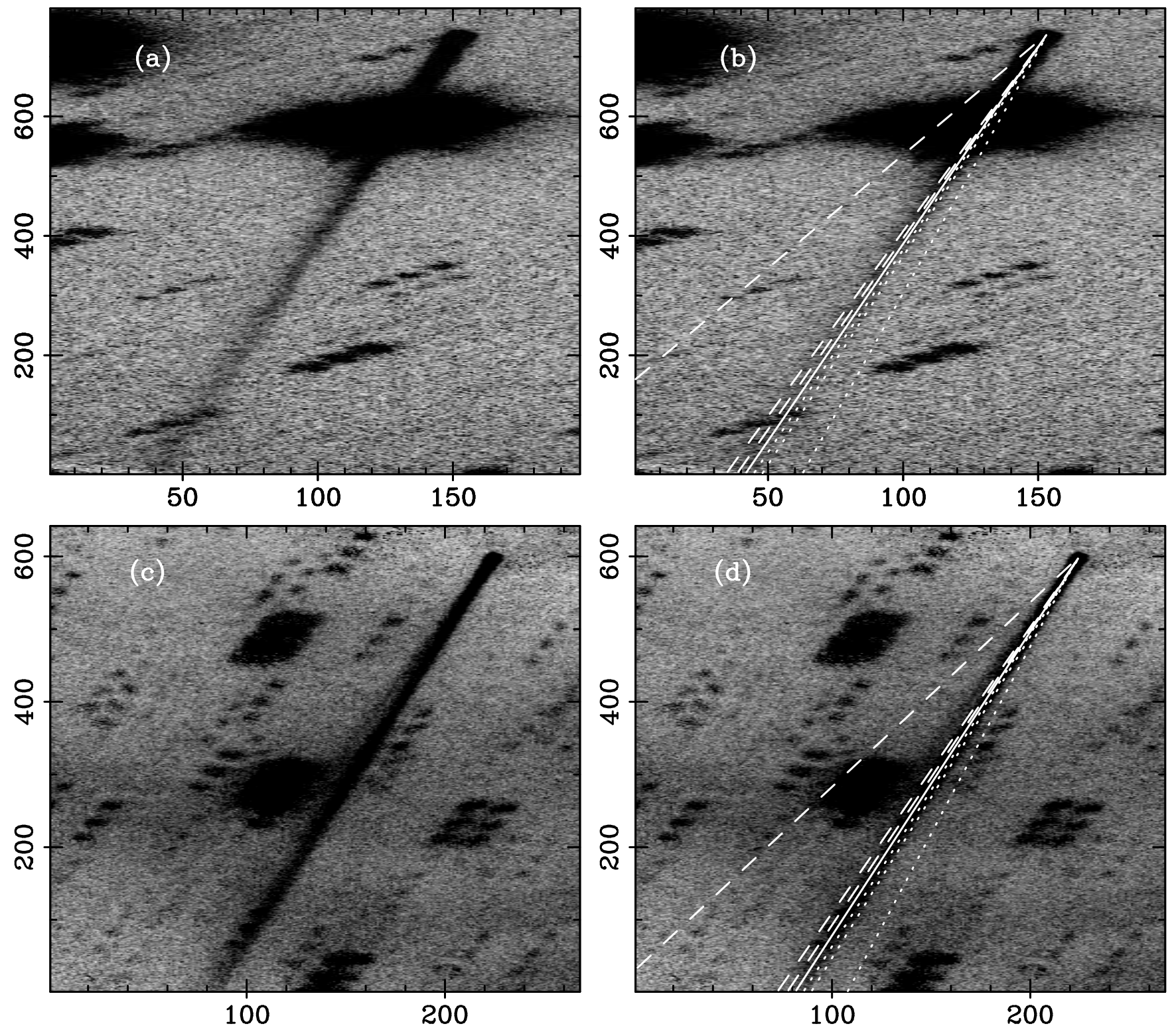

Fig. 2.- Panel(a) displays the P/2012 F5 (Gibbs) image obtained in 2012 May 18.9. Panel (b) shows the same image but with seven synchrones overlaid, corresponding, in clockwise order, to $-100,-20,-10$ (dotted lines), 0 (solid line), $+10,+20$, and +100 (dashed lines) days respect to the 2011 July 3 synchrone. Panels (c) and (d) shows the same as (a) and (b), respectively, but for the 2012 June 8.9 image. The scale is $485 \mathrm{~km} \mathrm{px}^{-1}$, and $540 \mathrm{~km}$ $\mathrm{px}^{-1}$, in the upper and lower panels, respectively. Note that the $x$ and $y$ axes are scaled independently to facilitate comparison between synchrones. 

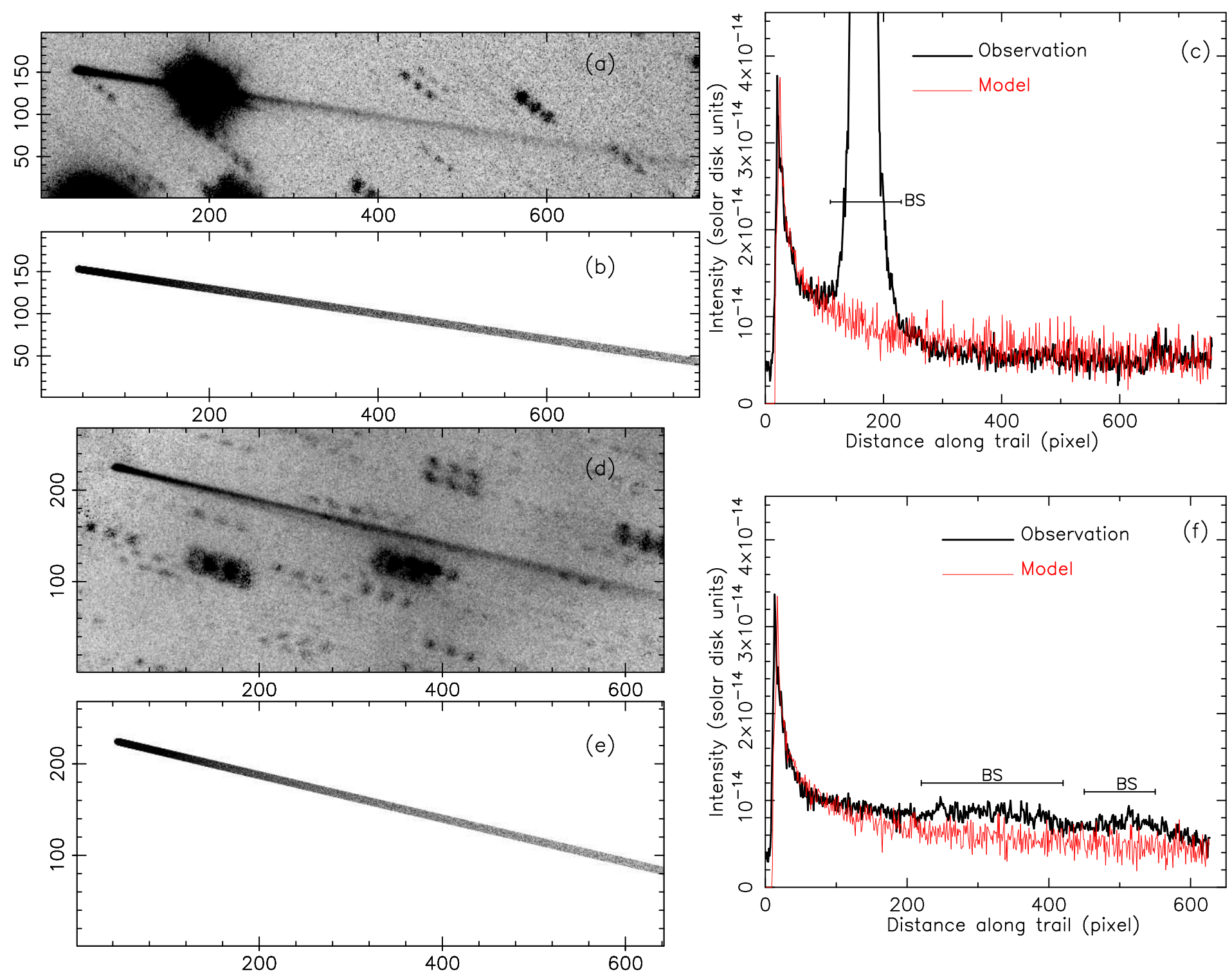

Fig. 3.- Panels (a) and (b) correspond to the observation and best fitted image for the observation on 2012 May 18th. Panel (c) shows observed and modeled scans along the trail of those images. Panels (d), (e), and (f) give the same as (a), (b), and (c), respectively, but for the 2012 June 8th image. The spatial scales are $485 \mathrm{~km} \mathrm{px}^{-1}$, and $540 \mathrm{~km} \mathrm{px}^{-1}$, respectively. Note that the images are rotated by 90 degree counterclockwise so that their $x$ and $y$ axes correspond to the photographic $M$ and $N$ axes. The segments labeled as "BS" pertain to regions of field star contamination in the trails. 
Table 1. The best-fit parameters of the model.

\begin{tabular}{ccccccc}
\hline \hline $\begin{array}{c}v_{0} \\
\left(\mathrm{~cm} \mathrm{~s}^{-1}\right)\end{array}$ & $\begin{array}{c}\text { Velocity } \\
\text { index }(\gamma)\end{array}$ & $\begin{array}{c}\text { SD power } \\
\text { index }(\alpha)\end{array}$ & $\begin{array}{c}\text { Max. radius } \\
\left(r_{\max }, \mathrm{cm}\right)\end{array}$ & $\begin{array}{c}\text { Mass lower } \\
\operatorname{limit}\left(M_{e}, \mathrm{~kg}\right)\end{array}$ & $\begin{array}{c}\text { Event } \\
\text { time }\left(t_{s}, \mathrm{UT}\right)\end{array}$ & $\begin{array}{c}\text { Event } \\
\text { duration }(\Delta t)\end{array}$ \\
\hline $13_{-8}^{+5}$ & $0.04_{-0.02}^{+0.06}$ & $-3.7 \pm 0.1$ & $28 \pm 10$ & $(5 \pm 2) \times 10^{8}$ & $2011 / 07 / 01 \pm 10 \mathrm{~d}$ & $0.13 \mathrm{~d}(<1 \mathrm{~d})$ \\
\hline
\end{tabular}

